\title{
Estudo comparativo da evolução nutricional de pacientes candidatos à cirurgia bariátrica assistidos pelo Sistema Único de Saúde e pela Rede Suplementar de Saúde
}

\author{
Comparative study of the nutritional evolution \\ of patients/candidates for bariatric surgery attended \\ by the Unified Health System and the Supplemental Health Network
}

${ }^{1}$ Universidade Federal de Sergipe (UFS). Av. Marechal Rondon $\mathrm{s} / \mathrm{n}$, Jardim Rosa Elze. 49.100-000 São Cristóvão SE Brasil. helis1935@hotmail.com

${ }^{2}$ Núcleo de Nutrição, UFS.

${ }^{3}$ Serviço de Nutrição e

Dietética, Hospital Universitário de Sergipe, UFS.

\begin{abstract}
A cross-sectional comparative study was conducted on the nutritional evolution of severely obese patients who are candidates for bariatric surgery attended by the Unified Health System (SUS) and the Supplemental Health Network (RS). The average preoperative follow-up period was $14.3 \pm 7.7$ months in the SUS and $2.4 \pm 1.7$ months in the RS. There was a predominance of females among patients attended by the SUS (80.6\%) and by the RS (75.7\%). A significant reduction in weight, BMI and waist circumference $(W C)$ and total cholesterol values of patients of both health networks was observed. Patients attended by the SUS showed no significant decrease in LDL, Triglycerides and Fasting Glycemia, while patients assisted by the RS showed a significant reduction of these values. The greater weight loss among SUS patients is explained by the fact that they had been monitored for a longer period. The insignificant reduction of most biochemical indicators of SUS patients can be justified by the fact that they had a more advanced degree of obesity.

Key words Obesity, Bariatric surgery, Nutritional status
\end{abstract}

Resumo Foi realizado um estudo transversal comparativo sobre a evolução nutricional de pacientes obesos graves candidatos à cirurgia bariátrica assistidos pelo Sistema Único de Saúde (SUS) e a Rede Suplementar de Saúde (RS). O tempo médio de acompanhamento pré-operatório foi $14,3 \pm 7,7$ meses no SUS e 2,4 $\pm 1,7$ meses na RS. Houve predominância feminina entre os pacientes assistidos pelo SUS (80,6\%) e pela RS (75,7\%). Foi observada uma redução significativa do peso, do IMC e na Circunferência da Cintura (CC) $e$ nos valores de Colesterol Total dos pacientes de ambas as redes de saúde. Os pacientes assistidos pelo SUS não apresentaram diminuição significativa nos valores de LDL, Triglicérides e Glicemia de Jejum, enquanto que os pacientes assistidos pela RS apresentaram uma redução significativa desses valores. A maior perda ponderal entre os pacientes do SUS explica-se pelos mesmos terem sido acompanhados por um tempo mais longo. A redução insignificante da maioria dos indicadores bioquímicos dos pacientes do SUS pode ser justificada pelos mesmos apresentarem um grau mais avançado da obesidade.

Palavras-chave Obesidade, Cirurgia bariátrica, Estado nutricional 


\section{Introdução}

A obesidade tornou-se um problema de saúde pública mundial e afeta indivíduos de todas as classes sociais ${ }^{1-3}$. Esse quadro é preocupante porque existe grande associação entre o excesso de gordura corporal e a elevação das taxas de morbi-mortalidade, uma vez que, essa condição aumenta o risco de desenvolvimento de doenças coronarianas, hipertensão arterial, diabetes mellitus tipo II, doença pulmonar obstrutiva, osteoartrite, certos tipos de câncer, dentre outras ${ }^{4,5}$.

As comorbidades relacionadas à obesidade, por serem patologias de longa duração, possuem uma grande demanda por ações, procedimentos e serviços de saúde, o que sobrecarrega o Sistema Único de Saúde (SUS) brasileiro, além da assistência prestada pelos serviços privados de saúde a $20 \%$ da população brasileira ${ }^{6,7}$.

O tratamento convencional dessa patologia envolve a orientação dietética, a prática de atividade física, a terapia cognitivo-comportamental e o uso de fármacos antiobesidade ${ }^{8}$. Quando esse procedimento não gera os efeitos esperados em obesos graves, é indicada a intervenção cirúrgi$\mathrm{ca}^{9,10}$. A cirurgia bariátrica é uma importante opção terapêutica para a obesidade grave, uma vez que induz o paciente a uma eficiente perda de peso e promove redução das comorbidades associadas a essa enfermidade ${ }^{11}$.

A atuação do nutricionista dentro da equipe multidisciplinar no período pré-operatório de cirurgia bariátrica, objetiva aumentar o potencial de sucesso no pós-operatório, a partir da preparação clínica e nutricional do paciente para a realização da mesma ${ }^{12,13}$.

O tempo médio de espera dos pacientes para a realização desta cirurgia, pelo SUS e pela Rede Suplementar de Saúde, é diferente. No SUS é de 2,9 anos $^{14}$, enquanto que pacientes que possuem planos de saúde privados conseguem realizá-la com o mínimo intervalo de tempo desde sua indicação, devendo-se apenas atender aos requisitos clínicos de realização da mesma ${ }^{15}$.

Considerando os impactos que essa divergência de tempo pode causar sobre o estado nutricional e, consequentemente, sobre a evolução clínica de indivíduos candidatos ao tratamento cirúrgico, o presente estudo pretende comparar a evolução nutricional de pacientes em pré-operatório de cirurgia bariátrica assistidos pelo SUS e pela Rede Suplementar de Saúde.

\section{Material e métodos}

Estudo transversal realizado com pacientes obesos graves em acompanhamento nutricional préoperatório de cirurgia bariátrica, assistidos pela mesma equipe de profissionais da saúde, no SUS (ambulatório de nutrição em cirurgia bariátrica do Hospital Universitário de Sergipe) e na Rede Suplementar de Saúde (consultórios de nutrição situados em clínicas particulares), ambos localizados na cidade de Aracaju, Sergipe.

Fizeram parte da amostra pacientes de ambos os sexos, com idade entre 21 e 63 anos, classificados como obesos grau II e grau III, de acordo com a Organização Mundial de Saúde (OMS), candidatos à cirurgia bariátrica.

Foram excluídos do estudo pacientes que não se encontravam no período pré-operatório de cirurgia bariátrica nos serviços selecionados ou que não apresentaram os dados completos, necessários à pesquisa.

Os dados foram coletados durante o período de janeiro a julho de 2012, nos protocolos de nutrição dos pacientes da amostra, em dois momentos diferentes: inicial (primeira consulta) e final (última consulta), antes da cirurgia. Os dados coletados foram: sexo, idade, datas da primeira e da última consulta antes da cirurgia, peso, altura, índice de massa corporal (IMC), circunferência da cintura, presença de comorbidades associadas à obesidade e exames bioquímicos.

A análise estatística dos dados foi realizada através do cálculo de média, desvio padrão e frequência. Foi utilizado para a comparação dos dados o teste paramétrico " $\mathrm{t}$ " pareado.

Foi considerado o nível de significância estatística de 5\% (p<0,05). As análises estatísticas foram realizadas utilizando o Statistical Package for the Social Science, SPSS versão 17.0 para o Windows.

O estudo foi aprovado pelo Comitê de Ética em Pesquisa com Seres Humanos da Universidade Federal de Sergipe, Brasil.

\section{Resultados}

Foram avaliados 73 pacientes, sendo 36 assistidos pelo Sistema Único de Saúde (SUS) e 37 acompanhados pela Rede Suplementar de Saúde (RS).

A maioria dos pacientes de ambos os serviços era do gênero feminino, sendo 80,6\% assistidas pelo SUS e 75,7\% pela RS (Gráfico 1). 
As comorbidades mais frequentes entre os pacientes foram semelhantes entre os assistidos pelo SUS e pela RS, sendo as mais prevalentes, a hipertensão arterial sistêmica, a dislipidemia e a esteatose hepática (Tabela 1).

Houve uma diferença expressiva no tempo de espera pelo procedimento cirúrgico entre os serviços de saúde estudados, sendo a média no SUS (14,3 $\pm 7,7$ meses) significativamente maior ( $\mathrm{p}=0,00)$ que na RS $(2,4 \pm 1,7$ meses).

Tanto os pacientes acompanhados pelo SUS (Tabela 2) quanto pela RS (Tabela 3) apresentaram redução significativa nas medidas de peso e circunferência da cintura, assim como nos valo-

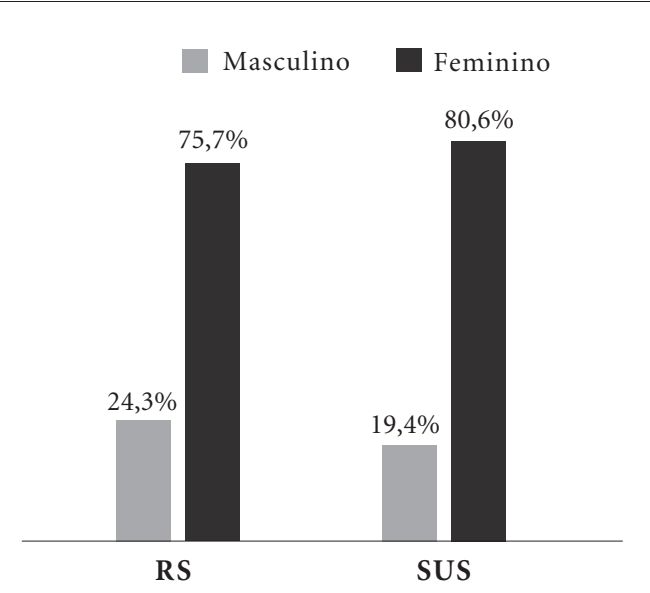

Gráfico 1. Distribuição por sexo dos pacientes da Rede Suplementar e do SUS.

Fonte: Hospital Universitário de Sergipe, 2012.

Tabela 1. Comorbidades mais frequentes entre os pacientes da Rede Suplementar de Saúde (RS) e do Sistema Único de Saúde (SUS), Aracaju-SE.

\begin{tabular}{lrrrrrr}
\hline \multirow{2}{*}{ Variáveis } & \multicolumn{2}{c}{ RS } & & \multicolumn{2}{c}{ SUS } \\
\cline { 2 - 3 } \cline { 6 - 7 } & N & \% & & N & $\%$ \\
\hline Hipertensão & 21 & 56,8 & & 28 & 77,8 \\
Arterial Sistêmica & & & & & \\
Dislipidemia & 17 & 45,1 & & 15 & 41,7 \\
Esteatose hepática & 10 & 27,0 & & 8 & 22,2 \\
Diabetes mellitus & 4 & 10,8 & & 13 & 36,1 \\
Cardiopatias & 2 & 5,4 & & 3 & 8,3 \\
Gastrite & 1 & 2,7 & & 13 & 36,1 \\
\hline
\end{tabular}

$\mathrm{n}=$ número amostral, \% frequência relativa.

Fonte: Hospital Universitário de Sergipe, 2012. res de IMC. Antes da intervenção nutricional, os dados antropométricos revelaram um quadro de superobesidade $\left(53,2 \pm 13,8 \mathrm{~kg} / \mathrm{m}^{2}\right)$ para os pacientes do SUS, enquanto os da RS apresentaram obesidade grau III $\left(40,2 \pm 4,5 \mathrm{~kg} / \mathrm{m}^{2}\right)$. Após a mesma, o estado nutricional da população do SUS era de obesidade grau III $\left(48,6 \pm 9,3 \mathrm{~kg} / \mathrm{m}^{2}\right)$ e o dos pacientes da RS era de obesidade grau II $\left(38,3 \pm 4,1 \mathrm{~kg} / \mathrm{m}^{2}\right)$ (Tabelas 2 e 3$)$.

Durante a assistência nutricional pré-operatória os participantes acompanhados pela RS (Tabela 3) mostraram redução significativa nos valores de LDL $(p=0,00)$, CT $(p=0,00), T G(p$

Tabela 2. Evolução nutricional dos pacientes candidatos à cirurgia bariátrica pelo Sistema Único de Saúde (SUS), Aracaju-SE.

\begin{tabular}{lrrr}
\hline \multirow{2}{*}{ Parâmetros } & \multicolumn{2}{c}{ SUS } & \\
\cline { 2 - 3 } & \multicolumn{1}{c}{ Inicial } & \multicolumn{1}{c}{ Final } & \\
& média \pm DP & média \pm DP & p $^{*}$ \\
\hline Peso $(\mathrm{kg})$ & $132,0 \pm 26,9$ & $123,2 \pm 30,0$ & 0,00 \\
IMC $\left(\mathrm{kg} / \mathrm{m}^{2}\right)$ & $53,2 \pm 13,8$ & $48,6 \pm 9,3$ & 0,01 \\
CC $(\mathrm{Cm})$ & $130,6 \pm 15,9$ & $124,8 \pm 16,0$ & 0,00 \\
HDL $(\mathrm{mg} / \mathrm{dL})$ & $46,3 \pm 12,0$ & $46,8 \pm 10,9$ & 0,78 \\
LDL $(\mathrm{mg} / \mathrm{dL})$ & $118,2 \pm 23,8$ & $108,4 \pm 27,7$ & 0,07 \\
CT $(\mathrm{mg} / \mathrm{dL})$ & $195,8 \pm 27,1$ & $181,7 \pm 30,9$ & 0,00 \\
TG $(\mathrm{mg} / \mathrm{dL})$ & $141,4 \pm 7,3$ & $134,2 \pm 7,7$ & 0,59 \\
Glic. JJ $(\mathrm{mg} / \mathrm{dL})$ & $101,6 \pm 28,0$ & $96,7 \pm 38,0$ & 0,46
\end{tabular}

* Teste “t” pareado $(\mathrm{p}<0,05)$

Fonte: Hospital Universitário de Sergipe, 2012.

Tabela 3. Evolução nutricional dos pacientes candidatos à cirurgia bariátrica pela Rede Suplementar de Saúde (RS), Aracaju-SE.

\begin{tabular}{lrrr}
\hline \multirow{2}{*}{ Parâmetros } & \multicolumn{2}{c}{ RS } & \\
\cline { 2 - 3 } & \multicolumn{1}{c}{ Inicial } & \multicolumn{1}{c}{ Final } \\
& & \\
\hline Peso $(\mathrm{kg})$ & $110,3 \pm 21,2$ & $105,0 \pm 19,2$ & 0,00 \\
IMC $\left(\mathrm{kg} / \mathrm{m}^{2}\right)$ & $40,2 \pm 4,5$ & $38,3 \pm 4,1$ & 0,00 \\
CC $(\mathrm{Cm})$ & $112,5 \pm 11,3$ & $109,0 \pm 10,1$ & 0,00 \\
HDL $(\mathrm{mg} / \mathrm{dL})$ & $42,4 \pm 9,2$ & $43,4 \pm 8,8$ & 0,50 \\
LDL $(\mathrm{mg} / \mathrm{dL})$ & $141,5 \pm 45,3$ & $107,8 \pm 23,6$ & 0,00 \\
CT $(\mathrm{mg} / \mathrm{dL})$ & $217,1 \pm 82,9$ & $177,4 \pm 44,0$ & 0,00 \\
TG $(\mathrm{mg} / \mathrm{dL})$ & $151,7 \pm 73,8$ & $131,6 \pm 58,9$ & 0,03 \\
Glic. JJ (mg/dL) & $106,0 \pm 46,2$ & $92,3 \pm 22,1$ & 0,01 \\
\hline
\end{tabular}

* Teste "t" pareado $(\mathrm{p}<0,05)$

Fonte: Hospital Universitário de Sergipe, 2012. 
$=0,03)$ e $\mathrm{GJ}(\mathrm{p}=0,01)$, enquanto os pacientes assistidos pelo SUS (Tabela 2) apresentaram redução significativa apenas no valor de CT $(\mathrm{p}=$ $0,00)$. Não houve aumento significativo entre os valores inicial e final de HDL dos pacientes assistidos por ambas redes de saúde (Tabelas 2 e 3 ).

Antes da intervenção nutricional, os pacientes acompanhados pelo SUS revelaram valores maiores de peso $(p=0,00)$, IMC $(p=0,00)$ e circunferência da cintura $(p=0,0)$ em relação aos assistidos pela RS. Quanto aos parâmetros bioquímicos, apenas o LDL apresentou diferença significativa $(\mathrm{p}=0,00)$ entre os pacientes dos dois âmbitos de saúde no momento inicial do estudo, sendo que os pacientes da RS apresentaram valor médio maior desse indicador (Tabela 4).

Após a intervenção nutricional, houve diferença significativa entre os valores antropométricos apresentados pelos pacientes das duas redes de saúde, sendo que os assistidos pelo SUS mostraram valores superiores. Entretanto, não houve diferença significativa entre os parâmetros bioquímicos dos pacientes acompanhados pelo SUS e pela RS (Tabela 4).

\section{Discussão}

A maior prevalência de mulheres em tratamento nutricional pré-operatório de cirurgia bariátrica pode ser explicada pela maior ocorrência da obesidade entre elas ou pelas diferenças de procura pelo tratamento ${ }^{11}$. De acordo com alguns autores, pode ser justificada também pela maior pre- ocupação desse público com a estética, com o preconceito que sofre por ser obeso e com a qualidade de vida ${ }^{16-18}$.

A alta prevalência das comorbidades entre a população estudada pode ser justificada pelo fato dessas doenças estarem associadas à obesidade grave $^{19}$. Estudos indicam que a hipertensão arterial sistêmica é a comorbidade mais prevalente entre os obesos ${ }^{20-22}$. Nas investigações de Souza ${ }^{18}$ foi observada também a liderança da hipertensão arterial sistêmica entre as comorbidades presentes, sendo a mesma seguida de dislipidemia.

A obesidade causa desordens metabólicas que induzem a ativação do Sistema Nervoso Simpático (SNS) e do Sistema Renina Angiotensina Aldosterona (SRAA) que estão relacionados com o controle da pressão arterial ${ }^{23}$. Estudos evidenciam que, uma vez ativado, o SNS ativa o SRAA o qual contribui para o desenvolvimento da hipertensão arterial devido aos efeitos pressóricos decorrentes da ação da angiotensina II sobre o tônus vascular e da aldosterona sobre a retenção de sódio no organismo ${ }^{24,25}$.

A grande quantidade de tecido adiposo apresentada pelos obesos causa um aumento exacerbado na taxa de lipólise que eleva as concentrações plasmáticas de ácidos graxos não esterificados e de algumas lipoproteínas, o que caracteriza a dislipidemia ${ }^{26}$.

Os valores iniciais de IMC revelaram um quadro de superobesidade para os pacientes do SUS e de obesidade grau III nos pacientes da RS.

Estudos realizados por Germano et al. ${ }^{27}$ e por Santos et al. ${ }^{28}$ com pacientes dos ambulatórios

Tabela 4. Comparação da evolução nutricional entre os pacientes da Rede Suplementar (RS) e do Sistema Único de Saúde (SUS), Aracaju-SE.

\begin{tabular}{|c|c|c|c|c|c|c|}
\hline \multirow[b]{3}{*}{ Parâmetros } & \multicolumn{3}{|c|}{ Inicial } & \multicolumn{3}{|c|}{ Final } \\
\hline & RS & SUS & & RS & SUS & \\
\hline & média $\pm \mathrm{DP}$ & média $\pm \mathrm{DP}$ & $\mathbf{p}^{*}$ & média $\pm \mathrm{DP}$ & média $\pm \mathrm{DP}$ & $\mathbf{p}^{*}$ \\
\hline Peso (kg) & $110,3 \pm 21,2$ & $132,0 \pm 26,9$ & 0,00 & $105,0 \pm 19,2$ & $123,2 \pm 30,0$ & 0,00 \\
\hline $\operatorname{IMC}\left(\mathrm{kg} / \mathrm{m}^{2}\right)$ & $40,2 \pm 4,5$ & $53,2 \pm 13,8$ & 0,00 & $38,3 \pm 4,1$ & $48,6 \pm 9,3$ & 0,00 \\
\hline $\mathrm{CC}(\mathrm{Cm})$ & $112,5 \pm 11,3$ & $130,6 \pm 15,9$ & 0,00 & $109,0 \pm 10,1$ & $124,8 \pm 16,0$ & 0,00 \\
\hline $\mathrm{HDL}(\mathrm{mg} / \mathrm{dL})$ & $42,4 \pm 9,2$ & $46,3 \pm 12,0$ & 0,13 & $43,4 \pm 8,8$ & $46,8 \pm 10,9$ & 0,16 \\
\hline LDL (mg/dL) & $141,5 \pm 45,3$ & $118,2 \pm 23,8$ & 0,00 & $107,8 \pm 23,6$ & $108,4 \pm 27,7$ & 0,97 \\
\hline $\mathrm{CT}(\mathrm{mg} / \mathrm{dL})$ & $217,1 \pm 82,9$ & $195,8 \pm 27,1$ & 0,12 & $177,4 \pm 44,0$ & $181,7 \pm 30,9$ & 0,71 \\
\hline $\mathrm{TG}(\mathrm{mg} / \mathrm{dL})$ & $151,7 \pm 73,8$ & $141,4 \pm 7,3$ & 0,46 & $131,6 \pm 58,9$ & $134,2 \pm 7,7$ & 0,95 \\
\hline Glic. JJ (mg/dL) & $106,0 \pm 46,2$ & $101,6 \pm 28,0$ & 0,58 & $92,3 \pm 22,1$ & $96,7 \pm 38,0$ & 0,61 \\
\hline
\end{tabular}

* Teste “t” pareado $(\mathrm{p}<0,05)$ 
de dois hospitais universitários do nordeste encontraram resultados parecidos ao do presente estudo. Entretanto, Moreira et al. ${ }^{21}$ avaliaram pacientes do ambulatório de cirurgia bariátrica do Hospital Universitário Oswaldo Cruz, em Pernambuco, e obtiveram como resultado do estado nutricional pré-operatório, obesidade grau III.

Avaliações realizadas com pacientes atendidos em consultórios privados de nutrição no Brasil verificaram que o estado nutricional dessa população era compatível com obesidade grau III, resultado semelhante ao deste estudo ${ }^{29-31}$.

O fato dos pacientes do SUS iniciarem a terapia nutricional com parâmetros antropométricos superiores aos dos assistidos pela Rede $\mathrm{Su}$ plementar pode ser explicado pela dificuldade de acesso aos serviços públicos de saúde por aquela população. O estudo de Moscon e Krüger ${ }^{32}$ respalda essa afirmação mostrando alguns fatores que dificultam o acesso, como: a demora do agendamento de consulta com especialistas na atenção secundária, serviços inviabilizados por infraestrutura ou equipamentos sucateados e falta de alguns profissionais nas equipes multidisciplinares. Além disso, os pacientes procuram esses serviços quando apresentam graus mais avançados de obesidade, uma vez que a consciência do tratamento ainda predomina sobre a da prevenção e, segundo Moscon e Krüger ${ }^{32}$, a população brasileira não tem o costume de buscar os serviços clínicos da atenção básica.

É na assistência de alta complexidade que o sistema público de saúde parece mais se aproximar da efetiva consecução do princípio da universalização do acesso, afirma estudo desenvolvido pelo Ministério da Saúde ${ }^{33}$.

A população do SUS apresentou uma perda de peso maior em relação à da RS. Isso se justifica pelo fato de serem acompanhados por um período de tempo maior, pois demoram a conseguir a liberação da cirurgia, visto as dificuldades encontradas neste serviço, tais como: marcação e liberação de alguns exames, limitação dos leitos hospitalares e greves em Hospitais da rede SUS ${ }^{34}$.

Houve diminuição significativa nos valores de LDL, TG e GJ dos participantes acompanhados pela RS, entretanto, esse resultado não foi encontrado na população do SUS.

Pesquisas indicam que a melhora do perfil metabólico de pacientes obesos causa diminui- ção da resistência insulínica e do risco de eventos coronarianos ateroscleróticos e de doenças cardiovasculares em geral, que podem influenciar negativamente o resultado perioperatório ${ }^{35-37}$.

A comparação de tais achados com outras pesquisas torna-se limitada em função da maioria dos estudos avaliarem a evolução nutricional entre os períodos pré e pós-operatório, e não apenas a evolução no momento pré-operatório, como proposto no presente estudo ${ }^{38-42}$.

\section{Conclusões}

A evolução nutricional da amostra populacional reflete a importância do acompanhamento nutricional pré-operatório de pacientes candidatos à cirurgia bariátrica, uma vez que a melhora dos parâmetros antropométricos e bioquímicos pode reduzir complicações no perioperatório.

A maior redução dos valores antropométricos dos pacientes assistidos pelo SUS, durante o tratamento nutricional, explica-se pelos mesmos terem sido acompanhados por um período de tempo mais longo. O fato dos pacientes do SUS não ter mostrado redução significativa na maioria dos indicadores bioquímicos pode ser justificado pelos mesmos apresentarem um grau mais avançado da obesidade, o que dificulta uma melhora significativa do perfil metabólico.

Sugere-se que novos estudos avaliem o impacto do tempo de espera pelo procedimento cirúrgico sobre a evolução nutricional de pacientes candidatos à cirurgia bariátrica para verificar a correlação entre ambos.

\section{Colaboradores}

HN Santos, JMS Lima e MFC Souza participaram igualmente de todas as etapas de elaboração do artigo. 


\section{Referências}

1. Wanderley EM; Ferreira VA. Obesidade: uma perspectiva plural. Cien Saude Colet 2010; 15(1):185 194.

2. Lima LP, Sampaio HAC. Caracterização socioeconômica, antropométrica e alimentar de obesos graves. Cien Saude Colet 2007; 12(4):1011-1020.

3. Moreira P, Romualdo MCS, Amparo FC, Paiva C, Alves R, Magnoni D, Kovacs C. A educação nutricional em grupo e sua efetividade em tratamento de pacientes obesos. Revista Brasileira de Obesidade, Nutrição e Emagrecimento 2012; 6(35):216-224.

4. Lemke GMMN, Correia JSC. Tratamento cirúrgico da obesidade e a ocorrência da Síndrome de Dumping. Saber Científico 2008; 1(1):176-193.

5. Sarturi JB, Neves J, Peres KG. Obesidade em adultos: estudo de base populacional num município de pequeno porte no sul do Brasil em 2005. Cien Saude Colet 2010; 15(1):105-113.

6. Coutinho JG, Gentil PC, Toral NA. Desnutrição e obesidade no Brasil: o enfrentamento com base na agenda única da nutrição. Cad Saude Publica 2008; 24(2):332-340.

7. Süssenbach SP. Custo Orçamentário da Cirurgia Bariátrica [dissertação]. Porto Alegre: Pontifícia Universidade Católica do Rio Grande do Sul; 2011.

8. Marcelino LF, Patrício ZM. A complexidade da obesidade e o processo de viver após a cirurgia bariátrica: uma questão de saúde coletiva. Cien Saude Colet 2011; 16(12):4767-4776.

9. Murara JR, Macedo LLB, Liberali R. Análise da eficácia da cirurgia bariátrica na redução de peso corporal e no combate à obesidade mórbida. Revista Brasileira de Obesidade, Nutrição e Emagrecimento 2008; 2(7):87-99.

10. Ribas Filho D, David IMB, Pacini JF, Miksche LC, Campos EMB, Moraes JC, Rias MG, Miquelin GM, Pereira DG. Avaliação de níveis lipêmicos e glicêmicos pré e pós cirurgia bariátrica. Rev Bras Clin Med 2009; 7(4):205-210.

11. Diniz MFHS, Passos VMA, Barreto SM, Diniz MTC, Linares DB, Mendes LN. Perfil de pacientes obesos classe III do Sistema Público de Saúde submetidos à gastroplastia em "Y de ROUX", no Hospital das Clínicas da UFMG: altas prevalências de superobesidade, co-morbidades e mortalidade hospitalar. Revista Médica de Minas Gerais 2008; 18(3):183-190.

12. Bregion NO, Silva SA, Salvo VLMA. Estado nutricional e condição de saúde de2 pacientes nos períodos pré e pós-operatórios de cirurgia bariátrica. Rev Brasileira de Ciências da Saúde 2007; (14):33-42.
13. Ravelli MN, Merhi VAL, Mônaco DV, Aranha N. Obesidade, cirurgia bariátrica e implicações nutricionais. Revista Brasileira em Promoção da Saúde 2007; 20(4):259-266.

14. Zilberstein B, Halpern A, Monteiro SM, Lunardi A, Campoleone S, Souza AC. Waiting time for bariatric surgery in a public hospital in Brazil: a problem to be solved. Obes Surg 2006; 16:1023.

15. Kelles SMB. Cirurgia bariátrica: mortalidade, utilização de serviços de saúde e custos. Estudo de caso em uma grande operadora do sistema de saúde suplementar no Brasil [dissertação]. Belo Horizonte: Universidade Federal de Minas Gerais; 2009.

16. Guedes AC, Virgens AA, Nascimento CE, Vieira, MPB. Qualidade de vida em pacientes submetidos à cirurgia bariátrica do tipo derivação biliopancreática com preservação gástrica (DBPPG). Rev Inst Ciênc Saúde 2009; 27(3):209-213.

17. Pereira JM, Buriola AA. Expectativa de jovens obesos frente à realização de cirurgia bariátrica. Colloquium Vitae 2011; 3(No Esp.):129-135.

18. Souza NPP. Evolução da obesidade da infância até a vida adulta entre mulheres da fila de espera para a cirurgia bariátrica pelo Sistema Único de Saúde [dissertação]. São Paulo: Universidade Estadual Paulista; 2007.

19. Marcon ER, Gus I, Neumann CR. Impacto de um programa mínimo de exercícios físicos supervisionados no risco cardiometabólico de pacientes com obesidade mórbida. Arq Bras Endocrinol Metab 2011; 55(5):331-338.

20. Costa ACC, Ivo ML, Cantero WB. Obesidade em pacientes candidatos a cirurgia bariátrica. Acta Paul Enferm 2009; 22(1):55-59.

21. Moreira MA, Silva SA, Araújo CMS, Nascimento CCC. Avaliação clínico-nutricional de obesos submetidos ao bypass gástrico em Y de Roux. Acta Gastroenterológica Latinoamericana 2010; 40(3):244-250.

22. Rocha IEGM, Victor EG, Braga MC, Silva OB, Becker MMC. Avaliação ecocardiográfica em obesos graves assintomáticos. Arq Bras Cardiol 2007; 88(1):52-58.

23. Lobato NS, Akamine EH, Tostes RC, Carvalho MHC, Fortes, ZB. Obesidade e hipertensão arterial. In: Anais do XVII Congresso da Sociedade Brasileira de Hipertensão; 2009; p. 4-12.

24. Lopes HF. Hipertensão e inflamação: papel da obesidade. Rev Brasileira de Hipertensão 2007; 14(4):239244. 
25. Fonseca-Alaniz MH, Krieger JE. Obesidade, hipertensão e sistema renina-angiotensina: aspectos moleculares de integração. In: Anais do XVII Congresso da Sociedade Brasileira de Hipertensão; 2009; p. 26-32.

26. Cheik NC, Guerra RLF, Viana FP, Rossi EA, Carlos IZ, Vendramini R, Duarte ACGO, Dâmaso AR. Efeito de diferentes freqüências de exercício físico na prevenção da dislipidemia e da obesidade em ratos normo e hipercolesterolêmicos. Rev. bras. Educ. Fís. Esp. 2006; 20(2):121-129.

27. Germano ACPL, Camelo CMBM, Batista FM, Carvalho NMA, Liberali R, Coutinho VF. Perfil nutricional dos pacientes submetidos à cirurgia bariátrica e dos critérios adotados para encaminhamento em um hospital de João Pessoa, PB. Ensaios e Ciência: Ciências Biológicas, Agrárias e da Saúde 2011; 14(2):43-59.

28. Santos EMC, Burgos MGPA, Silva SA. Perda ponderal após cirurgia bariátrica de Fobi-Capella: realidade de um hospital universitário do nordeste brasileiro. Rev Bras Nutr Clin 2006; 21(3):188-192.

29. Cruz MRR, Morimoto IMI. Nutritional intervention in the surgical treatment for morbid obesity: results of a differentiated protocol. Rev Nutr 2004; 17(2):263-272.

30. Mônaco DV, Merhi VAL, Aranha N, Brandalise A, Brandalise NA. Impact of bariatric surgery "modified capella type" on ponderal loss in patients with morbid obesity. Rev. Ciênc. Méd. 2006; 15(4):289298.

31. Prevedello CF, Colpo E, Mayer E T, Copetti H. Análise do impacto da cirurgia bariátrica em uma população do centro do estado do Rio Grande do Sul utilizando o método Baros. Arq Gastroenterol 2009; 46(3):199-203.

32. Moscon N, Krüger TR. O serviço social na atenção básica e o acesso aos serviços de saúde. Rev Saude Publica 2010; 3(2):88-98.

33. Instituto de Pesquisa Econômica Aplicada (IPEA). Projeto Economia da Saúde. Atenção de alta complexidade no SUS: desigualdades no acesso e no financiamento. Brasília: IPEA; 2005.

34. Diniz MFHS. Aspectos clínicos e metabólicos de pacientes obesos do Sistema Único de Saúde, submetidos à operação de Capella no hospital das clínicas da UFMG [tese]. Belo Horizonte: Universidade Federal de Minas Gerais; 2007.
35. Pereira JS. Perfil glicêmico e lipídico pré e pós-cirurgia bariátrica [monografia]. Salvador: Faculdade de Medicina da Bahia; 2012.

36. Monteiro Júnior FC, Silva Júnior WS, Salgado Filho N, Ferreira PAM, Araújo GF, Mandarino NR, Barbosa JB, Lages JS, Lima JRO, Monteiro CC. Efeito da perda ponderal induzida pela cirurgia bariátrica sobre a prevalência de síndrome metabólica. Arq Bras Cardiol 2009; 92(6):452-456.

37. Auler Júnior JOC, Giannini CG, Saragiotto DF. Desafios no manuseio perioperatório de pacientes obesos mórbidos: como prevenir complicações. Rev Bras Anestesiol 2003; 53(2):227-236.

38. Pedrosa IV, Burgos MGPA, Souza NC, Morais CN. Nutrition aspects in obese before and after bariatric surgery. Rev. Col. Bras. Cir. 2009; 36(4):316-322.

39. Nassif PAN, Lopes AD, Lopes GL, Martins PR, Pedri LE, Varaschim M, Bopp DS. Changes in the pre and postoperative parameters in metabolic syndrome patients submitted to roux-en-y gastric bypass. Arq Bras Cir Dig 2009; 22(3):165-170.

40. Lima-Filho JA, Ganem EM, Cerqueira BGP. Reavaliação da via aérea do paciente obeso submetido à cirurgia bariátrica após a redução do índice de massa corpórea. Rev Bras Anestesiol 2011; 61(1):31-40.

41. Carvalho PS, Moreira CLCB, Barelli MC, Oliveira FH, Guzzo MF, Miguel GPS, Zandonade E. Cirurgia Bariátrica Cura Síndrome Metabólica? Arq Bras Endocrinol Metab 2007; 51(1):79-85.

42. Vicente MA, Freitas AR. Avaliação das alterações nutricionais, clínicas e bioquímicas em pacientes submetidos à cirurgia bariátrica nos períodos pré e pós-cirúrgico. Revista Brasileira de Obesidade, Nutrição e Emagrecimento 2011; 5(28):333-343.

Artigo apresentado em 15/03/2013

Aprovado em 07/04/2013

Versão final apresentada em 15/04/2013 